\title{
CONTROLLABILITY OF GENERALISED DYNAMICAL SYSTEMS WITH CONSTRAINED CONTROL
}

\author{
CHEN ZHAOLIN, HONG HUIMIN, AND ZHANG JIFENG ${ }^{1}$
}

(Received 16 January 1987; revised 2 September 1987)

\begin{abstract}
The state controllability for generalised dynamical systems with constrained control is discussed in this paper. The main results of the paper are the following:

(1) a necessary and sufficient condition of the state controllability in the sense of control energy or amplitude constrained for generalised dynamical systems is obtained;

(2) a control function $u(t)$ is constructed such that

a) $u(t)$ satisfies constrained energy or amplitude condition,

b) the state driven by $u(t)$ moves from an arbitrary $x\left(0^{-}\right)=x_{0}$ to $x\left(T\left(x_{0}\right)\right)=$ 0 ,

c) the trajectory driven by $u(t)$ has no impulsive behaviour within $\left(0, T\left(x_{0}\right)\right]$.
\end{abstract}

\section{Introduction}

A generalised dynamical system can be described by

$$
E \dot{x}=A x+B u,
$$

where $x \in R^{n}, u \in R^{r}, E, A \in R^{n \times n}, B \in R^{n \times r}, E, A$ and $B$ are constant matrices, $E$ is singular, $E$ and $A$ satisfy $\operatorname{det}[s E-A] \not \equiv 0$. Earlier works on generalised dynamical systems were by Rosenbrock [6], [7] and Luenberger [4], who mainly discussed the decomposition of systems and the structure of solutions. Later, Verghese [8] and others introduced concepts of strong controllability, strong observability and testing criteria. Then Cobb [1], [2] searched for state feedback, pole assignment, optimal regulation, and so on. Some literature has indicated that generalised dynamical systems can be extensively applied to

\footnotetext{
${ }^{1}$ Shandong University, Jinan, P.R.C.

(C) Copyright Australian Mathematical Society 1988, Serial-fee code 0334-2700/88
} 
network and socio-economical systems (Rosenbrock [6], [7], Luenberger and Arbel [5]). Discussions developed in those papers, however, were mostly for systems with unconstrained control. It seems to the present authors that the problem of constrained control should be considered in many cases. In this paper, a problem of state controllability for generalised dynamical systems with constrained control is emphasised. A constrained-control function is constructed which leads to the state travelling from arbitrary $x\left(0^{-}\right)=x_{0}$ to $x\left(T\left(x_{0}\right)\right)=0$, and is such that the trajectory has no impulsive behaviour within $\left(0, T\left(x_{0}\right)\right]$.

\section{State controllability for generalised dynamical systems with constrained control}

System (1.1) can always be restricted system equivalent (RSE) to a system described by Rosenbrock:

$$
\Sigma:\left\{\begin{array}{l}
\dot{x}_{1}=A_{1} x_{1}+B_{1} u \\
N \dot{x}_{2}=x_{2}+B_{2} u,
\end{array}\right.
$$

where $x_{1} \in R^{n_{1}}, x_{2} \in R^{n_{2}}, n_{1}+n_{2}=n, u \in R^{r}, A_{1}, B_{1}, B_{2}$ and $N$ are constant matrices with suitable orders, $N$ is nilpotent and its index of nilpotency is $\nu$. RSE means there exist nonsingular $P, Q \in R^{n \times n}$ such that

$$
\begin{aligned}
& P(s E-A) Q=\left[\begin{array}{ll}
s I_{n_{1}}-A_{1} & \\
& s N-I_{n_{2}}
\end{array}\right], \\
& P B=\left[\begin{array}{l}
B_{1} \\
B_{2}
\end{array}\right] \text {. }
\end{aligned}
$$

Obviously, RSE can be constructed by two transformations:

i) applying a row transformation $P$ of full rank to system (1.1);

ii) applying a full rank transformation $Q$ to the state $x$ of system (1.1).

It is clear that the above transformations do not affect the dynamical structure of system (1.1). Therefore, we can consider the state controllability of system $\Sigma$ instead. System $\Sigma$ comprises two subsystems which are not coupled. The linear system (2.1) is called the slow subsystem and (2.2) whose natural response contains impulsive behaviour is called the fast subsystem.

DEFINITION 2.1. For a given generalised dynamical system (1.1) with control energy (or control amplitude) constrained by $L$, a state $x_{0}$ is controllable in the sense of control energy (or control amplitude) constrained, if there exist a finite $T\left(x_{0}\right)$ and a control $u(t)$, such that

$$
\int_{0}^{T\left(x_{0}\right)} u^{\tau} u d t \leq L
$$


or

$$
\|u\|=\sqrt{\sum_{i=1}^{r} u_{i}^{2}} \leq L,
$$

where $\left(u_{1} u_{2} \ldots u_{r}\right)^{\tau}=u$, and $u(t)$ leads the state moving from $x\left(0^{-}\right)=x_{0}$ to $x\left(T\left(x_{0}\right)\right)=0$, and the trajectory $x(t)$ has no impulsive behaviour within $\left(0, T\left(x_{0}\right)\right]$. System (1.1) is called state controllable, in the sense of control energy (or control amplitude) constrained, if any state is controllable in the sense of control energy (or control amplitude) constrained.

LEMMA 2.1. [9] Let

$$
W_{k}=I+C \bar{C}^{\tau}+\cdots+C^{k-1}\left(\bar{C}^{k-1}\right)^{\tau}
$$

where $C \in C^{n \times n}$, and $\bar{C}^{\top}$ is conjugate transposed matrix of $C$. Then

$$
W_{k}^{-1} \rightarrow 0 \quad \text { as } k \rightarrow \infty
$$

if and only if all eigenvalues of $C$ do not lie inside the unit circle, i.e. $\left|\lambda_{j}(C)\right| \geq 1$, $j=1,2, \ldots, n$.

LEMMA 2.2. [9] A linear system described by

$$
\dot{x}=A x+B u
$$

is state controllable in the sense of control energy (or control amplitude) constrained, if and only if

i) $\operatorname{rank}\left[B A B \ldots A^{n-1} B\right]=n$, where $n$ is the dimension of the state;

ii) none of the eigenvalues of $A$ has a positive real part.

This lemma shows that for linear systems, the state controllability subjected to constraint (2.5) or (2.6) is equivalent to conditions i) and ii) in the lemma.

LEMMA 2.3, If system (2.1) is state controllable, let

$$
f_{m, n_{0}}(t)= \begin{cases}t^{m}(1-t)^{m} & t \in[0,1] \\ (t-1)^{m}(2-t)^{m} & t \in[1,2] \\ \vdots & \\ \left(t-n_{0}+1\right)^{m}\left(n_{0}-t\right)^{m} & t \in\left[n_{0}-1, n_{0}\right],\end{cases}
$$

where $m, n_{0}$ are natural numbers. Then the matrix defined by

$$
W\left(f_{m, n_{0}}, 0, n_{0}\right)=\int_{0}^{n_{0}} f_{m, n_{0}}^{2}(t)\left[\exp \left(-A_{1} t\right)\right] B_{1} B_{1}^{\tau}\left[\exp \left(-A_{1} t\right)\right]^{\tau} d t
$$


is positive definite, and there exist positive $\alpha_{i}(m), \beta_{i}(m), i=1,2$, such that

$$
\begin{aligned}
\alpha_{1}(m) W\left(f_{m, n_{0}}, 0, n_{0}\right) & \leq W\left(f_{2 m, n_{0}}, 0, n_{0}\right) \leq \beta_{1}(m) W\left(f_{m, n_{0}}, 0, n_{0}\right) \\
\alpha_{2}(m) W\left(n_{0}\right) & \leq W\left(f_{m, n_{0}}, 0, n_{0}\right) \leq \beta_{2}(m) W\left(n_{0}\right)
\end{aligned}
$$

where

$$
W\left(n_{0}\right)=\sum_{i=0}^{n_{0}-1}\left[\exp \left(-A_{1} i\right)\right]\left[\exp \left(-A_{1} i\right)\right]^{\tau}
$$

ProOF. Omitted.

If a generalised dynamical system $\Sigma$ is described by (2.1) and (2.2), the initial state is $x\left(0^{-}\right)=\left[\begin{array}{ll}x_{10}^{\tau} & x_{20}^{\tau}\end{array}\right]^{\tau}$, the control $u$ has up to $(\nu-1)$ continuous derivatives, and

$$
u(0)=u^{(1)}(0)=\cdots=u^{(\nu-1)}(0)=0,
$$

where $u^{(i)}(\cdot)$ denotes the $i$ th derivative, then the solution of system $\Sigma$ is of the form (Cobb [1]):

$$
\begin{aligned}
& x_{1}(t)=\exp \left(A_{1} t\right) x_{10}+\int_{0}^{t} \exp \left[A_{1}(t-s)\right] B_{1} u(s) d s, \\
& x_{2}(t)=-\sum_{i=0}^{\nu-2} N^{i+1} \delta^{(i)}(t) x_{20}-\sum_{i=0}^{\nu-1} N^{i} B_{2} u^{(i)}(t)
\end{aligned}
$$

where $\delta(t)$ is the Dirac- $\delta$ function. From (2.16), although $x_{2}(t)$ contains an impulse at $t=0$, it can be seen that the value of $x_{2}(t)$ only depends on $u^{(i)}(t)$, $i=0,1, \ldots, \nu-1$, as $t>0$. Therefore, $x(t)$ driven by $u(t)$ can be transferred from $x\left(0^{-}\right)=\left[x_{10}^{\tau} x_{20}^{\tau}\right]^{\tau}$ to $x\left(T\left(x_{0}\right)\right)=0$ without any impulsive behaviour within $\left(0, T\left(x_{0}\right)\right]$, provided that $u(t)$ leads $x_{1}(t)$ moving from $x_{10}$ to $x_{1}\left(T\left(x_{0}\right)\right)=0, u(t)$ is sufficiently smooth and $u^{(i)}(0)=0, u^{(i)}\left(T\left(x_{0}\right)\right)=0, i=0,1, \ldots, \nu-1$.

THEOREM 2.1. A generalised dynamical system (1.1) is state controllable in the sense of control energy constrained, if and only if its RSE system $\Sigma$ satisfies

i) the slow subsystem (2.1) is state controllable:

ii) the slow subsystem (2.1) has no eigenvalue whose real part is positive.

ProOF. Necessity. Since the state controllability of system $\Sigma$ in the sense of control energy constrained contains that of slow subsystem (2.1), applying Lemma 2.2 to the slow subsystem (2.1) gives the necessity.

Sufficiency. Let the initial state $x\left(0^{-}\right)=\left[\begin{array}{ll}x_{10}^{\tau} x_{20}^{\tau}\end{array}\right]^{\tau}$. The control function $u(t)$ can be constructed as the following:

$$
u(t)=-f_{\nu, n_{0}}^{2}(t) B_{1}^{\tau}\left[\exp \left(-A_{1} t\right)\right]^{\tau} W^{-1}\left(f_{\nu, n_{0}}, 0, n_{0}\right) x_{10},
$$


where $f_{\nu, n_{0}}(t)$ is defined as in (2.9), $\nu$ is the nilpotency index of matrix $N$, $W\left(f_{\nu, n_{0}}, 0, n_{0}\right)$ is defined as in (2.10) and $n_{0}$ is undetermined. It is clear that $u(t)$ has up to $(\nu-1)$ continuous derivatives, and satisfies

$$
u^{(i)}(0)=u^{(i)}(1)=\cdots=u^{(i)}\left(n_{0}\right)=0 \quad i=0,1, \ldots, \nu-1 .
$$

Hence, from (2.15) and (2.16), we know that $x\left(n_{0}\right)=0$, and $x(t)$ does not contain any impulsive behaviour within $\left(0, n_{0}\right]$, when system $\Sigma$ is driven by $u(t)$.

Now let us investigate the control energy. Without loss of generality, let $x_{10} \neq 0$. Notice that

$$
\begin{aligned}
& \int_{0}^{n_{0}} u^{\tau} u d t=x_{10}^{\tau} W^{-1}\left(f_{\nu, n_{0}}, 0, n_{0}\right) \cdot \int_{0}^{n_{0}} f_{\nu, n_{0}}^{4}(t) \exp \left(-A_{1} t\right) B_{1} B_{1}^{\tau}\left[\exp \left(-A_{1} t\right)\right]^{\tau} d t \\
& W^{-1}\left(f_{\nu, n_{0}}, 0, n_{0}\right) x_{10}
\end{aligned}
$$

and

$$
f_{\nu, n_{0}}^{2}(t)=f_{2 \nu, n_{0}}(t)
$$

Considering (2.11)-(2.13) and (2.19) $-(2.20)$ gives

$$
\begin{aligned}
\int_{0}^{n_{0}} u^{\tau} u d t & \leq \beta_{1}(\nu) x_{10}^{\tau} W^{-1}\left(f_{\nu, n_{0}}, 0, n_{0}\right) x_{10} \\
& \leq \beta_{1}(\nu) \operatorname{trace} W^{-1}\left(n_{0}\right)\left\|x_{10}\right\|^{2} / \alpha_{2}(\nu) .
\end{aligned}
$$

All eigenvalues of $\exp \left(-A_{1}\right)$ are obviously located on or outside the unit circle, since eigenvalues of $A_{1}$ have no positive real part. Applying Lemma 2.1 to (2.13), it follows that $W^{-1}\left(n_{0}\right) \rightarrow 0$, as $n_{0} \rightarrow \infty$. Equivalently, trace $W^{-1}\left(n_{0}\right) \rightarrow 0$, as $n_{0} \rightarrow \infty$. Therefore, for the given limited value $L$ of the control energy, we can determine a sufficiently large $n_{0}$, such that

$$
\operatorname{trace} W^{-1}\left(n_{0}\right) \leq \alpha_{2}(\nu) L /\left(\left\|x_{10}\right\|^{2} \beta_{1}(\nu)\right) .
$$

Thus

$$
\int_{0}^{n_{0}} u^{\tau} u d t \leq L
$$

that is, the energy of $u$ is constrained by $L$.

LEMMA 2.4. If system (2.1) is state controllable, let

$$
\begin{gathered}
W\left(m, T, n_{1}\right)=\sum_{k=0}^{n_{1}-1}\left\{\int_{k T}^{(k+1) T}(t-k T)^{m}((k+1) T-t)^{m} \exp \left(-A_{1} t\right) B_{1} d t\right\} \\
\cdot\left\{\int_{k T}^{(k+1) T}(t-k T)^{m}((k+1) T-t)^{m} \exp \left(-A_{1} t\right) B_{1} d t\right\}^{\tau}
\end{gathered}
$$

where $n_{1}$ is the dimension of the state of system (2.1), $m$ is a natural number, and $T>0$, and let

$$
e=e_{1} \cup e_{2},
$$


where

$$
\begin{gathered}
e_{1}=\left\{\begin{array}{l}
\theta: \theta=2 k \pi / \operatorname{Im}\left(\lambda_{i}-\lambda_{j}\right), k=0, \pm 1, \pm 2, \ldots, \\
\lambda_{i}, \lambda_{j} \text { are eigenvalues of } A_{1} \text { with different imaginary parts }
\end{array}\right\} \\
e_{2}=\bigcup_{m=1}^{\infty} e_{2}(m), \\
e_{2}(m)=\left\{\theta: \int_{0}^{\theta} t^{m}(\theta-t)^{m} \exp \left(\lambda_{j} t\right) d t=0, \lambda_{j} \text { is an eigenvalue of } A_{1}\right\},
\end{gathered}
$$

then $e$ is a denumerable set, and $W\left(m, T, n_{1}\right)$ is positive definite, as $T \in$ $(0, \infty)-e$.

ProOF. i) $e$ is denumerable.

$e_{1}$ is obviously denumerable, since $\sigma\left(A_{1}\right)$, the set of eigenvalues of $A_{1}$, is finite. Denote

$$
\psi_{m, j}(\theta)=\int_{0}^{\theta} t^{m}(\theta-t)^{m} \exp \left(\lambda_{j} t\right) d t
$$

Since

$$
d^{m+1} \psi_{m, j}(\theta) / d \theta^{m+1}=m ! \theta^{m} \exp \left(\lambda_{j} \theta\right)
$$

then $\psi_{m, j}(\theta)$ is of the form

$$
\psi_{m, j}(\theta)=\varphi_{1}(\theta) \exp \left(\lambda_{j} \theta\right)+\varphi_{2}(\theta)
$$

where $\varphi_{1}(\theta)$ and $\varphi_{2}(\theta)$ are polynomials with complex coefficients, and they are not identically vanishing. Equation (2.31) says that the set of zeros of $\psi_{m, j}(\theta)$ is at most denumerable. Therefore, $e_{2}(m)$ is denumerable, and so are $e_{2}$ and $e$.

ii) $W\left(m, T, n_{1}\right)$ is positive definite, as $T \in(0, \infty)-e$.

Let

$$
\begin{aligned}
G & =\exp \left(A_{1} T\right) \\
H_{0}(m) & =\int_{0}^{T} t^{m}(T-t)^{m} \exp \left(A_{1} t\right) d t \\
H(m) & =H_{0}(m) B_{1}
\end{aligned}
$$

Also, $W\left(m, T, n_{1}\right)$ can be rewritten in the form

$$
W\left(m, T, n_{1}\right)=\sum_{k=0}^{n_{1}-1} G^{-(k+1)} H(m) H^{\tau}(m)\left[G^{-(k+1)}\right]^{\tau}
$$

It is obvious that the state controllability of the linear discrete system $[G, H(m)]$ guarantees that $W\left(m, T, n_{1}\right)$ is positive definite. 
Now let us investigate the state controllability of $[G, H(m)]$. Noticing that the product of $H_{0}(m)$ and $G$ is commutative, we have

$$
\operatorname{rank}\left[H(m) G H(m) \ldots G^{n_{1}-1} H(m)\right]=\operatorname{rank} H_{0}(m)\left[B_{1} G B_{1} \ldots G^{n_{1}-1} B_{1}\right] .
$$

Since system (2.1) is state controllable and $T \in(0, \infty)-e$, the linear discrete system $\left[G, B_{1}\right]$ is state controllable (Guan and Chen [3]), that is,

$$
\operatorname{rank}\left[B_{1} G B_{1} \cdots G^{n_{1}-1} B_{1}\right]=n_{1} .
$$

From (2.27) and (2.28), and noticing that

$$
\begin{aligned}
\operatorname{det} H_{0}(m) & =\operatorname{det} \int_{0}^{T} t^{m}(T-t)^{m} \exp \left(A_{1} t\right) d t \\
& =\prod_{j=1}^{n_{1}} \int_{0}^{T} t^{m}(T-t)^{m} \exp \left(\lambda_{j} t\right) d t \quad \lambda_{j} \in \sigma\left(A_{1}\right),
\end{aligned}
$$

$H_{0}(m)$ is full rank, as $T \in(0, \infty)-e_{2}$. Thus, system $[G, H(m)]$ is state controllable, as $T \in(0, \infty)-e$, and $W\left(m, T, n_{1}\right)$ is positive definite, as $T \in(0, \infty)-e$. The lemma now has been proved.

LEMMA 2.5. Suppose system (2.1) is state controllable, $n_{1}$ is the dimension of the state of system (2.1), and $e$ is defined as in $(2.25)-(2.28), T \in(0, \infty)-e$. Let

$$
\begin{aligned}
& W(m, T, P)= \sum_{k=0}^{P-1}\left\{\int_{k T}^{(k+1) T}(t-k T)^{m}((k+1) T-t)^{m} \exp \left(-A_{1} t\right) B_{1} d t\right\} \\
& \cdot\left\{\int_{k T}^{(k+1) T}(t-k T)^{m}((k+1) T-t)^{m} \exp \left(-A_{1} t\right) B_{1} d t\right\} \\
& W(N, T)=\sum_{i=0}^{N-1} \exp \left(-A_{1} i n_{1} T\right)\left[\exp \left(-A_{1} i n_{1} T\right)\right]^{\tau}
\end{aligned}
$$

where $P=n_{1} N, N$ is a natural number. Then $W(m, T, P)$ is positive definite, and there exist $\alpha(m, T), \beta(m, T)>0$, such that

$$
\alpha(m, T) W(N, T) \leq W(m, T, P) \leq \beta(m, T) W(N, T) .
$$

PROOF. Omitted.

THEOREM 2.2. A generalised dynamical system (1.1) is state controllable in the sense of control amplitude constrained if and only if its RSE system $\Sigma$ 


\section{satisfies}

i) the slow subsystem (2.1) is state controllable;

ii) the slow subsystem (2.1) has no eigenvalue whose real part is positive.

ProOF. Sufficiency. Choose $T$ from $(0,1)-e$ arbitrarily, where $e$ is defined as in (2.25)-(2.28). Denote

$$
H=H(\nu)=\int_{0}^{T} t^{\nu}(T-t)^{\nu} \exp \left(A_{1} t\right) B_{1} d t
$$

where $\nu$ is the nilpotency index of matrix $N$. For the initial state $x\left(0^{-}\right)=$ $\left[\begin{array}{ll}x_{10}^{\tau} & x_{20}^{\tau}\end{array}\right]^{\tau}$, set the control function:

$$
\begin{aligned}
u(t) & = \begin{cases}u_{0}(t) & t \in[0, T] \\
u_{1}(t) & t \in[T, 2 T] \\
\vdots & \\
u_{P-1(t)} & t \in[(P-1) T, P T]\end{cases} \\
u_{k}(t) & =-(t-k T)^{\nu}((k+1) T-t)^{\nu} H^{\tau}\left\{\exp \left[-A_{1}(k+1) T\right]\right\}^{\tau} W^{-1}(\nu, T, P) x_{10}
\end{aligned}
$$

where $W(\nu, T, P)$ is defined as in (2.39), $P=n_{1} N$, and $N$ is undetermined. It is clear that $u(t)$ has up to $(\nu-1)$ continuous derivatives, and that

$$
u^{(i)}(0)=u^{(i)}(T)=\cdots=u^{(i)}(P T)=0 \quad i=0,1, \ldots, \nu-1 .
$$

Notice

$$
\begin{aligned}
\exp \left[-A_{1}(k+1) T\right] H & =\exp \left[-A_{1}(k+1) T\right] \int_{0}^{T} t^{\nu}(T-t)^{\nu} \exp \left[A_{1}(T-t) B_{1} d t\right. \\
& =\int_{k T}^{(k+1) T}(t-k T)^{\nu}((k+1) T-t)^{\nu} \exp \left(-A_{1} t\right) B_{1} d t
\end{aligned}
$$

$$
\sum_{k=0}^{P-1} \exp \left[-A_{1}(k+1) T\right] H H^{\tau}\left\{\exp \left[-A_{1}(k+1) T\right]\right\}^{\tau}=W(\nu, T, P),
$$

and the solution expressions (2.15) and (2.16) for the system $\Sigma$. Hence, driven by $u(t)$, the state $x(t)$ of system $\Sigma$ is zero at $t=P T$, and $x(t)$ does not contain any impulsive behaviour within $(0, P T]$.

Now we are in the position to estimate $u(t)$, as $t \in[0, P T]$. Without loss of generality, let $x_{10} \neq 0$. From (2.43) and (2.44), it is obvious that

$$
\begin{aligned}
\|u(t)\|^{2} & =\left\|u_{k}(t)\right\|^{2} \\
& =(t-k T)^{2 \nu}((k+1) T-t)^{2 \nu}\left\|H^{\tau}\left\{\exp \left[-A_{1}(k+1) T\right]\right\}^{\tau} W^{-1}(\nu, T, P) x_{10}\right\|^{2} \\
t & \in[k T,(k+1) T], \quad k=0,1, \ldots, P-1 . \quad(2.48)
\end{aligned}
$$


Since $T \in(0,1)-e$, we have

$$
(t-k T)^{2 \nu}((k+1) T-t)^{2 \nu} \leq(T / 2)^{4 \nu} \leq 1 .
$$

Therefore, from (2.47)-(2.49), and (2.41), we obtain

$$
\begin{aligned}
\|u(t)\|^{2} & \leq\left\|H^{\tau}\left\{\exp \left[-A_{1}(k+1) T\right]\right\}^{\tau} W^{-1}(\nu, T, P) x_{10}\right\|^{2} \\
& \leq \sum_{k=0}^{P-1}\left\|H^{\tau}\left\{\exp \left[-A_{1}(k+1) T\right]\right\}^{\tau} W^{-1}(\nu, T, P) x_{10}\right\|^{2} \\
& =x_{10}^{\tau} W^{-1}(\nu, T, P) x_{10} \leq \operatorname{trace} W^{-1}(\nu, T, P)\left\|x_{10}\right\|^{2} \\
& \leq \operatorname{trace} W^{-1}(N, T)\left\|x_{10}\right\|^{2} / \alpha(\nu, T) .
\end{aligned}
$$

Eigenvalues of $\exp \left(-A_{1} n_{1} T\right)$ are located on or outside the unit circle, since eigenvalues of $A_{1}$ have no positive real parts. Applying Lemma 2.1 to (2.40) gives trace $W^{-1}(N, T) \rightarrow 0$, as $N \rightarrow \infty$. Therefore, for the given limited value $L$ of control amplitude, we can determine a sufficiently large $N$, such that

$$
\operatorname{trace} W^{-1}(N, T) \leq L^{2} \alpha(\nu, T) /\left\|x_{10}\right\|^{2} .
$$

Thus

$$
\|u(t)\| \leq L \quad t \in[0, P T]
$$

that is the amplitude of $u$ is constrained by $L$. The sufficiency now has been proved.

The necessity is trivial.

\section{Acknowledgement}

This work was supported by the Science Fund of Chinese Academy of Sciences, and the Microcomputer Consulting and Service Centre of Jinan. The authors gratefully acknowledge this support.

\section{References}

[1] D. Cobb, "Feedback and pole placement in descriptor variable systems", Internat. J. Control 336 (1981) 1135-1146.

[2] D. Cobb, "Discriptor variable systems and optimal state regulation", IEEE Trans. AC-28 5 (1983) 601-611.

[3] Z. Guan and H. Chen, Controllability and observability of linear systems (Science Press, Beijing, China, 1975).

[4] D. Luenberger, "Dynamical equation in descriptor form", IEEE Trans. AC-22 3 (1977) 312-321.

[5] D. Luenberger and A. Arbel, "Singular dynamic Leontief systems", Econometrica (1977). 
[6] H. Rosenbrock, "Structural properties of linear dynamical systems", Internat. J. Control 20 (1974) 191-202.

[7] H. Rosenbrock, "Non-minimal LCR multiports", Internal. J. Control 20 (1974) 1-16.

[8] H. Verghese, B. Levy and T. Kailath, "A generalised state-space for singular systems, IEEE. Trans. AC-26 4 (1981) 811-831.

[9] K. Zhao, Z. Chen and Z. Cheng, "Complete controllability for linear constant systems with control constraints", Proc. 9th IFAC Congr. 3 (1984) 1415-1419. 